\title{
Influence of Addition of Alumina Nanoparticles on Thermoelectric Properties of La-Filled Skutterudite $\mathrm{CoSb}_{3}$ Compounds
}

\author{
Takashi Itoh and Masaki Matsuhara* \\ Department of Materials Science and Engineering, Nagoya University, Nagoya 464-8603, Japan
}

\begin{abstract}
The $\mathrm{CoSb}_{3}$-based compound is a promising thermoelectric material useful in the temperature range of $600-900 \mathrm{~K}$. It, however, has a higher thermal conductivity. Thus, reducing the thermal conductivity causes improvement of the thermoelectric performance. In this work, we tried to reduce the thermal conductivity by both filling La and dispersing alumina nanoparticles (ANPs) into the compound. The raw powders of LaSb, $\mathrm{Co}, \mathrm{Ni}, \mathrm{Sb}$ and Te were prepared. The n-type La-filled compound was synthesized from the raw powders using a planetary ball mill. Then, ANPs were added to the combined powder and further mixed using the planetary ball milling. The mixture was sintered by a pulse-discharge sintering method. The combining phases of the samples were characterized by X-ray diffraction analysis. The thermoelectric properties were measured for the samples. Influence of addition of ANPs on the properties was studied. Adding 0.05 mass $\%$ ANPs reduced the thermal conductivity. The sample added 0.5 mass $\%$ or more ANPs increased both the electrical resistivity and the thermal conductivity. As a result, the sample added 0.05 mass \% ANPs had the maximum performance of $Z T=1.15$ at $873 \mathrm{~K}$. [doi:10.2320/matertrans.E-M2012821]
\end{abstract}

(Received November 24, 2011; Accepted June 25, 2012; Published August 22, 2012)

Keywords: filled skutterudite compounds, addition of alumina nanoparticles, mechanical alloying, pulse discharge sintering, thermoelectric properties

\section{Introduction}

Since the world consumes a huge amount of energy, the exhaustion of fossil fuels and the impact on environment become serious issues. The thermoelectric power generation (TEG) that can directly convert thermal energy into electrical energy has advantages of quietness, compactness and low environmental impact. Thus, TEG using waste heat is a promising renewable energy system. However, TEG dose not sufficiently reach to the level of commercial and industrial use due to its low energy conversion efficiency.

The performance of the semiconductor is usually evaluated with the figure of merit $Z$, defined by

$$
Z \equiv \frac{\alpha^{2}}{\rho \kappa},
$$

where $\alpha$ is the Seebeck coefficient, $\rho$ the electrical resistivity, and $\kappa$ the thermal conductivity. The thermal conductivity appears in the denominator of $Z$, because the thermoelectric material act as a thermal insulator between the hot and the cold sides. ${ }^{1)}$ The reason that the electrical resistivity also appears in the denominator of $Z$ is due to the Joule heating in the element. The decrease in the electrical resistivity minimizes the Joule heat. Because a unit of $Z$ is reciprocal of temperature, the dimensionless figure of merit $Z T$ is often used. The $Z T$ value is strongly related to the conversion efficiency. Among three parameters in eq. (1), the Seebeck coefficient depends on the carrier concentration, and the electrical resistivity depends on the carrier concentration and the carrier mobility.

The thermal conductivity of thermoelectric semiconductors consists of contributions from electrons and phonons, with the major contribution coming from phonons. It is expressed with the following equation:

*Graduate Student, Nagoya University. Present address: Naoetsu Works, Sumitomo Metal Industries Ltd., Joetsu 942-8510, Japan

$$
\kappa=\kappa_{\mathrm{E}}+\kappa_{\mathrm{L}},
$$

where $\kappa_{\mathrm{E}}$ and $\kappa_{\mathrm{L}}$ are the electron and lattice thermal conductivities, respectively. According to the WiedemannFranz Law, the electron thermal conductivity is calculated as follows:

$$
\kappa_{\mathrm{E}}=\frac{L T}{\rho},
$$

where $L$ is the Lorenz number. The electron thermal conductivity also depends on the carrier concentration and the carrier mobility, because it directly depends on the electrical resistivity. The lattice thermal conductivity, however, does not depend on both the carrier concentration and the carrier mobility. By applying the phonon gas theory, the lattice thermal conductivity can be expressed with the following equation.

$$
\kappa_{\mathrm{L}}=\frac{1}{3} C_{\mathrm{v}} v l,
$$

where $C_{\mathrm{v}}$ is the heat capacity, $v$ the phonon velocity and $l$ the phonon mean free path. In a solid, the broad spectrum of phonon frequencies complicates the situation. Since the optical phonons with high energy do not have enough high group velocities to transport an appreciable quantity of heat, the longer-wavelength acoustic phonons frequently take the thermal transport. ${ }^{2)}$ Thus, the phonon velocity can correspond to the mean sound velocity. Since the phonon mean free path is the maximum distance the phonons can travel, it can be affected by various scattering events. Much of the focus has been on obtaining low thermal conductivity through the mechanisms discussed below: (a) disorder or distortion of unit cells, (b) resonant scattering by localized rattling atoms and (c) creation of a high density of interfaces. $^{3)}$

Skutterudite $\mathrm{CoSb}_{3}$ compound has better thermoelectric performance. However, the thermal conductivity is relatively 
high, and it prevents achievement of high thermoelectric performance. Uher has provided an in-depth review pertaining to the skutterudite materials. ${ }^{4)}$ Their lattice thermal conductivity is mainly lowered by filling atoms as "rattlers" into the interstitial sites of lattice in the skutterudite structure. ${ }^{5-11)}$ Reduction of the phonon mean free path by decreasing the grain size of skutterudite compound and by uniformly dispersing the nanoparticles into matrix of the compound could cause effective reduction of the lattice thermal conductivity, leading to improvement in thermoelectric properties. In our previous works, we have uniformly dispersed the fullerene particles, ${ }^{12)}$ the $\mathrm{ANPs}^{13)}$ and the carbon nanotubes $(\mathrm{CNTs})^{14)}$ into n-type skutterudite $\mathrm{CoSb}_{3}$ compounds. And we have reported that optimum addition of these nano-sized materials reduces the thermal conductivity and improves the thermoelectric performance $Z T$.

In this study, we tried to lower the thermal conductivity and improve the thermoelectric performance by both filling atoms and dispersing nanoparticles uniformly into n-type skutterudite $\mathrm{CoSb}_{3}$ compounds. We selected lanthanum as rattler atom, and nano-sized alumina as nanoparticles. In our previous study, the filling ratio of La was decided for getting the best thermoelectric performance of n-type $\mathrm{Co}_{3.68} \mathrm{Ni}_{0.32} \mathrm{Sb}_{11.84} \mathrm{Te}_{0.16}$ compound. Four kinds of law material powders were prepared for synthesizing the $\mathrm{La}_{0.3} \mathrm{Co}_{3.68} \mathrm{Ni}_{0.32} \mathrm{Sb}_{11.84} \mathrm{Te}_{0.16}$ compound. The synthesis of the compound was carried out by mechanical alloying (MA) using a planetary ball mill. After MA, the alumina nanoparticles (ANPs) were added to the combined powder and further mixed using a planetary ball milling. The mixed powder was sintered by a pulse discharge sintering (PDS). The thermoelectric properties of the sintered samples were measured. Influence of addition of ANPs on the thermoelectric properties was studied.

\section{Experimental Procedures}

The raw material powders of Co (purity: $99 \%$ or more, particle size: less than $75 \mu \mathrm{m}), \mathrm{Sb}(99.9 \%$ or more, less than $45 \mu \mathrm{m}), \mathrm{Ni}(99.9 \%$ or more, $2-3 \mu \mathrm{m}$.), Te (99.9\% or more, less than $53 \mu \mathrm{m})$ and $\mathrm{LaSb}(99.9 \%$ or more, less than $200 \mu \mathrm{m})$ were prepared for synthesizing the n-type compound. These powders are of Kojundo Chemical Lab. Co., Ltd., Japan. In order to promote the synthesizing reaction, the LaSb powder was ground at $400 \mathrm{rpm}$ for $10 \mathrm{~h}$ in an argon atmosphere using a planetary ball mill (P-6, Fritsch $\mathrm{GmbH}$, Germany). A milling pot of stainless steel (capacity: $250 \mathrm{cc}$ ) and 85 pieces of stainless steel balls (diameter: $9.5 \mathrm{~mm}$ ) were used for milling. The powder with particle size of less than $45 \mu \mathrm{m}$ was separated from the milled powder by sieving in an argon atmosphere.

The four kinds of raw material powders with stoichiometric mole ratio were weighed, and the $\mathrm{La}_{0.3} \mathrm{Co}_{3.68^{-}}$ $\mathrm{Ni}_{0.32} \mathrm{Sb}_{11.84} \mathrm{Te}_{0.16}$ compounds were synthesized by MA method using the planetary ball mill at $400 \mathrm{rpm}$ for $10 \mathrm{~h}$ in an argon atmosphere. Then, the $\gamma$-ANPs (mean particle size: $50 \mathrm{~nm}$, Praxair K.K., Japan) were added into the combined powder. For uniformly dispersing the ANPs, the mixture was mechanically ground using the planetary ball mill at $150 \mathrm{rpm}$ for $30 \mathrm{~min}$ in an argon atmosphere. The ground powder was collected and dried within a glove box in an argon atmosphere. The mixed powder samples with $0-2$ mass $\%$ of ANPs $(0,0.05,0.5$ and 2 mass $\%)$ were prepared. The mixed powder sample was packed in a cylindrical graphite container of $20 \mathrm{~mm}$ in diameter. Then, it was heated at $50 \mathrm{~K} \mathrm{~min}^{-1}$ and sintered at $873 \mathrm{~K}$ for $30 \mathrm{~min}$ in a vacuum under a pressure of $30 \mathrm{MPa}$ by PDS method. After sintering, the sample was cooled at $-1 \mathrm{~K} \mathrm{~min}^{-1}$ in a chamber of PDS apparatus (SPS-1050, SPS SYNTEX Inc., Japan).

The quadratic prism specimens $(3 \times 3 \times 15 \mathrm{~mm})$ were prepared from the sintered samples, and the Seebeck coefficient and electrical resistivity were measured with a thermoelectric measuring apparatus (ZEM-2, ULVAC-RIKO Inc., Japan). The disk-type specimens $(\phi 10 \times 1 \mathrm{~mm})$ were prepared from the sintered samples, and the thermal conductivity was measured with a laser-flash apparatus (TC-7000, ULVAC-RIKO Inc.). The synthesized phases of the sintered samples were identified using an X-ray diffraction (XRD) apparatus (RINT2500TTR, RIGAKU Corp., Japan). The XRD analysis was carried out in the diffraction angle range of 20 to 90 degree using characteristic $\mathrm{Cu} \mathrm{K} \alpha$ radiation. The microstructure of the sintered samples was also observed using a scanning electron microscopy with energy dispersive X-ray (SEM-EDX TypeN1, Hitachi High-Technologies Corp., Japan) in order to confirm the effect of grain refining and the dispersing state of ANPs.

\section{Results and Discussion}

We confirmed that all sintered samples had high relative density of $95 \%$ or more. The samples were sufficiently sintered by PDS. Figure 1 shows the results of X-ray diffraction analysis of the samples with different addition amount of ANPs. The single phase of the skutterudite $\mathrm{CoSb}_{3}$ compound was identified regardless of difference of addition amount of ANPs. This result indicates that the $\mathrm{La}_{0.3} \mathrm{Co}_{3.68^{-}}$ $\mathrm{Ni}_{0.32} \mathrm{Sb}_{11.84} \mathrm{Te}_{0.16}$ compound was successfully synthesized by MA-PDS method. The reason why the alumina phase was not identified would be due to small addition amount of ANPs and relatively weak intensity of alumina.

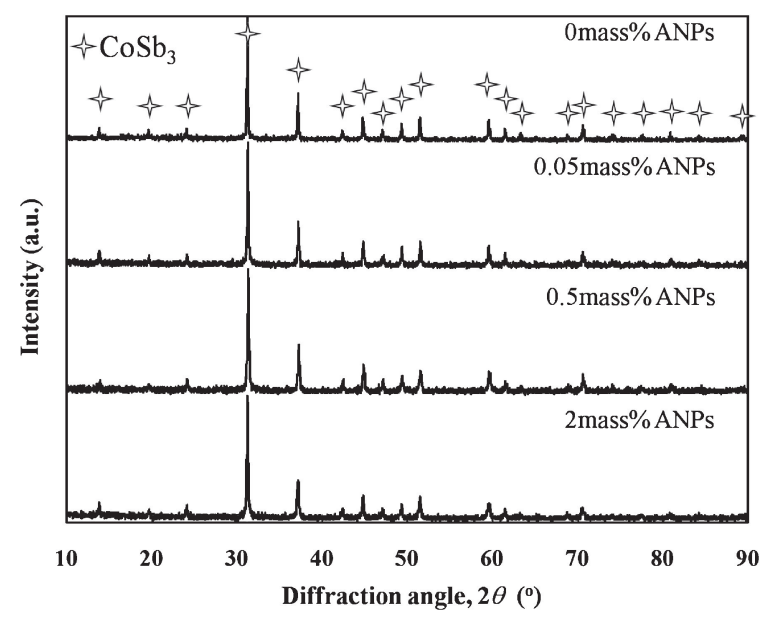

Fig. 1 X-ray diffraction patterns of samples with different addition amount of ANPs. 

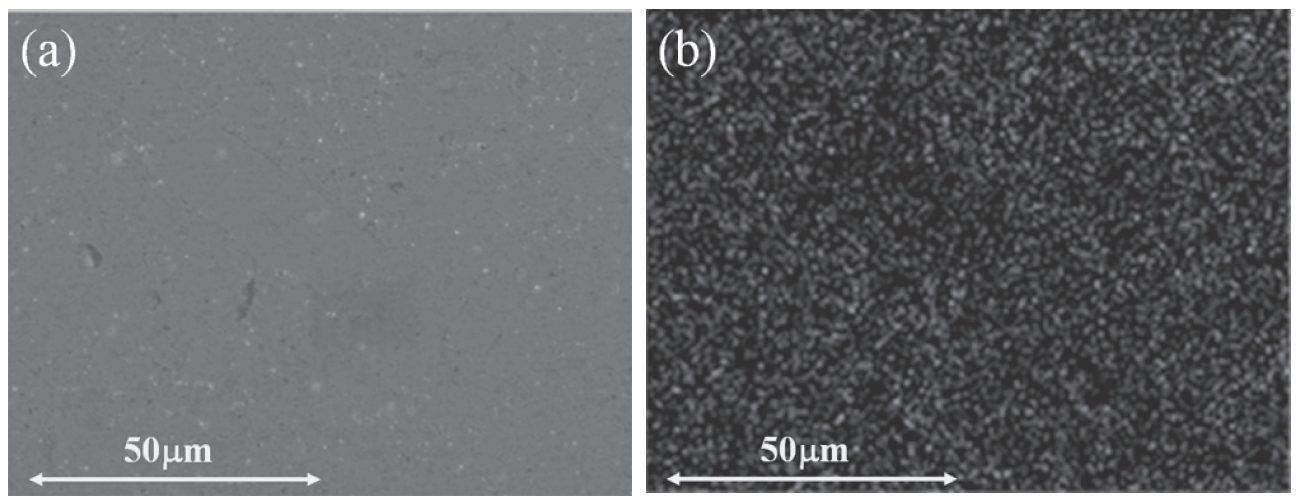

Fig. 2 Cross section images of sample added 2 mass\% ANPs: (a) SEM image and (b) aluminum mapping image obtained by EDX analysis.

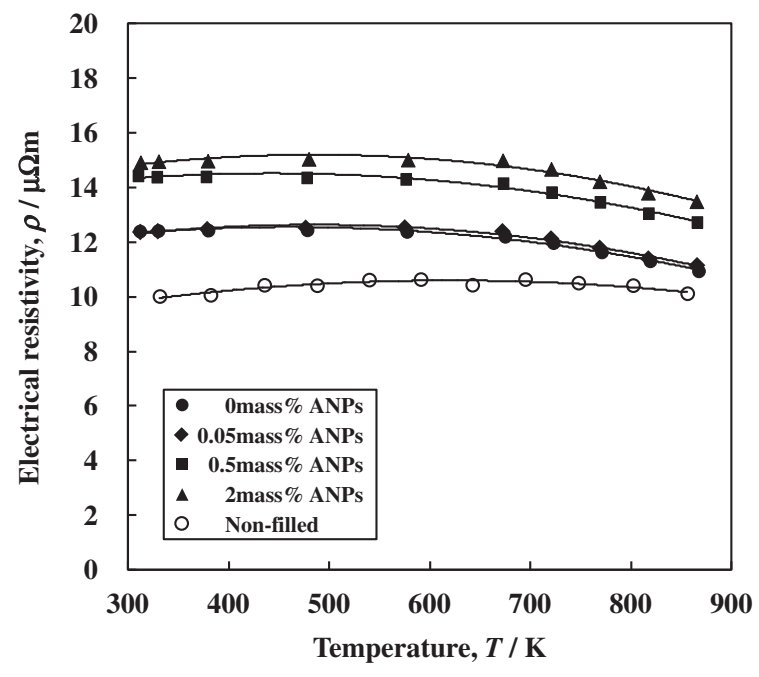

Fig. 3 Temperature dependences of electrical resistivity for different addition amount of ANPs together with data of non-filled sample.

Figure 2 shows (a) the SEM image and (b) the aluminum mapping image obtained by EDX analysis in a cross section of the sample added 2 mass $\%$ ANPs. The white dots in the mapping image indicate the location at which the aluminum atoms were detected, that is, the location of ANPs. Though existence of some aggregates of ANPs was confirmed in the skutterudite compound matrix, the aluminum mapping image indicates that the nanoparticles almost uniformly disperse in the matrix.

Figure 3 shows the temperature dependences of the electrical resistivity for the different addition amount of ANPs together with data of the non-filled sample $\left(\mathrm{Co}_{0.92-}\right.$ $\left.\mathrm{Ni}_{0.08} \mathrm{Sb}_{2.96} \mathrm{Te}_{0.04}\right)$ prepared in our previous work. ${ }^{12)}$ The electrical resistivity was increased by filling lanthanum. It would be caused by decrease in the carrier concentration. ${ }^{11)}$ The resistivity still more increased with increase of addition amount of ANPs. It is thought that the increase in resistivity by adding ANPs was caused by the high resistivity of alumina and the carrier scattering at the nanoparticles dispersed in the skutterudite compound matrix.

The temperature dependences of the Seebeck coefficient for the different addition amount of ANPs are shown in Fig. 4. The data of the non-filled sample ${ }^{12)}$ were also

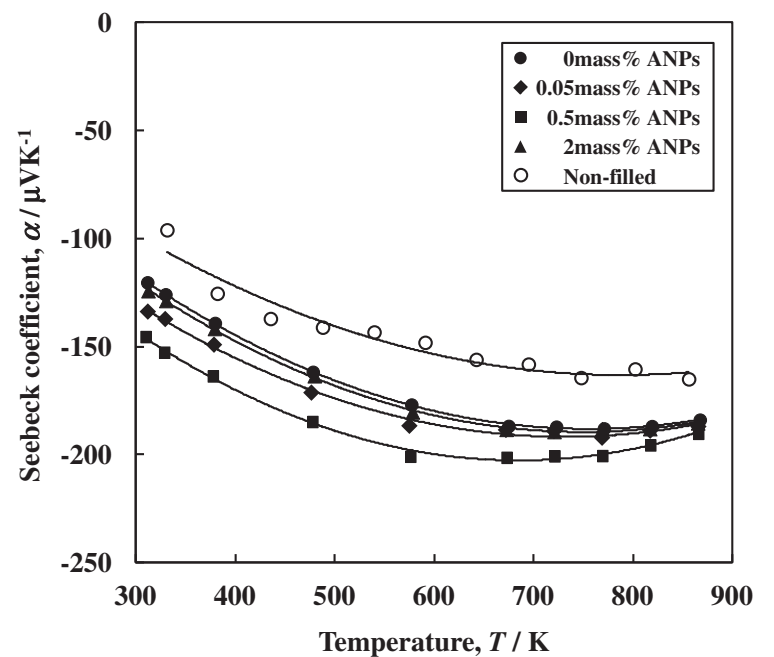

Fig. 4 Temperature dependences of Seebeck coefficient for different addition amount of ANPs together with data of non-filled sample.

represented in this figure. The Seebeck coefficients of all samples indicated n-type. By filling lanthanum, the absolute value of the Seebeck coefficient was increased. The decrease in the carrier concentration by filling lanthanum ${ }^{11)}$ would bring the increase in the absolute value. The Seebeck coefficient was hardly changed by addition of ANPs except the sample added 0.5 mass $\%$ ANPs. Though some notsystematized variation existed in the Seebeck coefficient, it should be thought that the Seebeck coefficient depending on the carrier concentration is hardly changed by addition of ANPs.

Let us consider the influence of addition of ANPs on the thermal conductivity. Figure 5 shows the temperature dependences of the thermal conductivity for the different addition amount of ANPs together with the data of the nonfilled sample. ${ }^{12)}$ The remarkable decrease in the thermal conductivity was caused by filling lanthanum. The phonon scattering based on rattling of filled atoms brought the large reduction in the thermal conductivity. In the addition of ANPs, the small addition amount decreased the thermal conductivity, but the surplus addition amount resulted in the re-increase in the conductivity. The lattice thermal conductivity was obtained by subtracting the electron thermal 


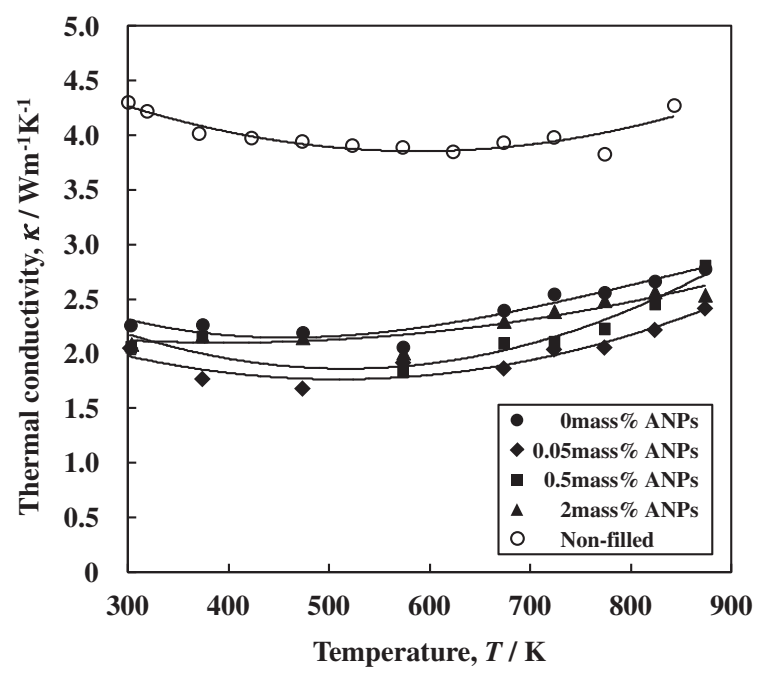

Fig. 5 Temperature dependences of thermal conductivity for different addition amount of ANPs together with data of non-filled sample.

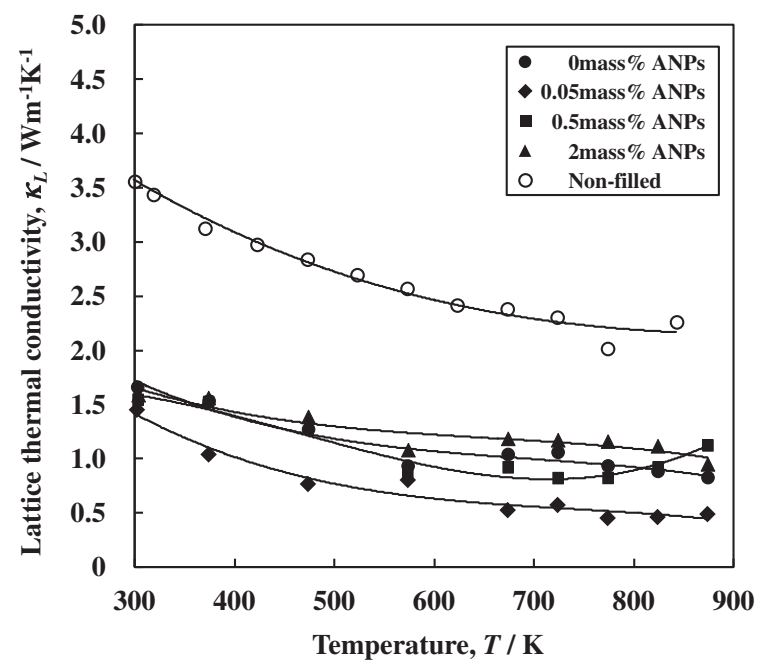

Fig. 6 Temperature dependences of lattice thermal conductivity for different addition amount of ANPs together with data of non-filled sample.

conductivity from the thermal conductivity measured. The electron thermal conductivity was calculated using eq. (3) and the Lorenz number of $2.45 \times 10^{-8} \mathrm{~V}^{2} \mathrm{~K}^{-2}$. The temperature dependences of the lattice thermal conductivity for the different addition amount of ANPs are shown in Fig. 6 together with data of the non-filled sample. ${ }^{12)}$ The variation in the lattice thermal conductivity by addition of ANPs was similar to that of the measured thermal conductivity. This indicates that the thermal conductivity of the La-filled skutterudite $\mathrm{CoSb}_{3}$ compound strongly depends on the behavior of phonon. In the small addition amount $(0.05 \mathrm{mass} \%)$, the lattice thermal conductivity was lowered by scattering phonon at the nanoparticles. However, in the surplus addition amount $(0.5$ mass $\%$ or more $)$, it became increased due to high thermal conductivity of alumina based on the compound rule.

In this research, the chemical composition of compound which is represented such as $\mathrm{La}_{0.3} \mathrm{Co}_{3.68} \mathrm{Ni}_{0.32} \mathrm{Sb}_{11.84} \mathrm{Te}_{0.16}$ is a composition in the preparation of synthesis, not an actual

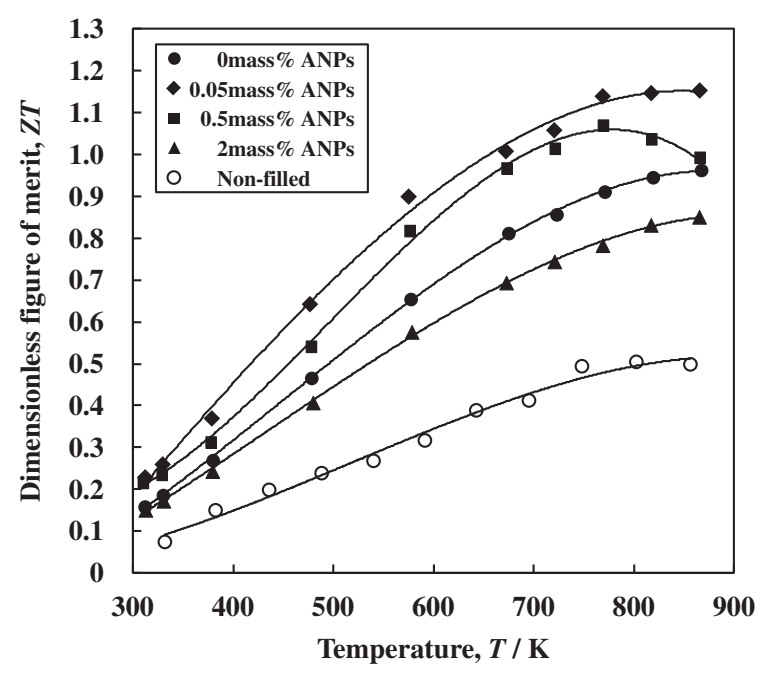

Fig. 7 Temperature dependences of $Z T$ for different addition amount of ANPs together with data of non-filled sample.

one. Since the thermoelectric properties of the skutterudite compounds are sensitively controlled by the chemical composition of compound or the carrier concentration, minute variation in the composition could bring the change in the properties. Thus, evaluation of the actual composition and the carrier concentration will be needed for more precisely investigating the influence of dispersion of the ANPs on the thermoelectric properties. The evaluation will be planned in our next research.

The dimensionless figure of merit $Z T$ is an index of thermoelectric performance, which closely relates to the conversion efficiency. This value was calculated using eq. (1). Figure 7 shows the temperature dependences of the dimensionless figure of merit for the different addition amount of ANPs together with data of the non-filled sample. ${ }^{12)}$ The value of $Z T$ was approximately doubled by filling lanthanum. By adding 0.05 mass $\%$ ANPs, the $Z T$ was still more increased. But the surplus addition amount ( 0.5 mass \% or more) brought the decrease in $Z T$. The sample added 0.05 mass $\%$ ANPs had the maximum thermoelectric performance of $Z T=1.15$ at $873 \mathrm{~K}$. It corresponds to the improvement of about $20 \%$ compared with the thermoelectric performance of the normal La-filled sample.

\section{Conclusion}

We tried to reduce the thermal conductivity and improve the thermoelectric performance of the n-type skutterudite $\mathrm{CoSb}_{3}$ by both filling lanthanum and adding ANPs using MA-PDS method. Addition of ANPs increased the electrical resistivity due to their high resistivity and the scattering of carriers at the dispersed nanoparticles. But the addition of ANPs hardly changed the Seebeck coefficient depending on the carrier concentration. Though small addition amount of ANPs decreased the thermal conductivity of the La-filled skutterudite $\mathrm{CoSb}_{3}$, the surplus addition of ANPs ( 0.5 mass\% or more) increased the thermal conductivity due to high thermal conductivity of alumina. Among all samples, the sample added 0.05 mass $\%$ ANPs had the maximum thermoelectric performance of $Z T=1.15$ at $873 \mathrm{~K}$. 


\section{REFERENCES}

1) G. Chen and A. Shakouri: J. Heat Transfer 124 (2002) 242-252.

2) E. S. Toberer, A. F. May and G. J. Snyder: Chem. Mater. 22 (2010) 624-634.

3) C. Wan, Y. Wang, N. Wang, W. Norimatsu, M. Kusunoki and K. Koumoto: Sci. Technol. Adv. Mater. 11 (2010) 044306 (7pp).

4) C. Uher: Semiconductors and Semimetals, Vol. 69, (Academic Press, San Diego, 2001) pp. 139-253.

5) B. C. Sales, D. Mandrus and R. K. Williams: Science 272 (1996) 1325 1328.

6) G. Mahan, B. Sales and J. Sharp: Phys. Today 50 (1997) 42-47.

7) B. C. Sales, D. Mandrus, B. C. Chakoumakos, V. Keppens and J. R. Thompson: Phys. Rev. B 56 (1997) 15081-15089.
8) G. S. Nolas, M. Kaeser, R. T. Littleton, IV and T. M. Tritt: Appl. Phys. Lett. 77 (2000) 1855-1857.

9) H. Anno, K. Ashida, K. Matsubara, G. S. Nolas, K. Akai, M. Matsuura and J. Nagao: Mater. Res. Soc. Symp. Proc., Vol. 691, (Cambridge University Press, 2001) pp. 49-54.

10) H. Anno, J. Nagao and K. Matsubara: Proc. 21st Int. Conf. on Thermoelectrics, (IEEE, New York, 2002) pp. 56-59.

11) T. Itoh, E. Hattori and K. Kitagawa: J. Propul. Power 24 (2008) 359364.

12) T. Itoh, K. Ishikawa and A. Okada: J. Mater. Res. 22 (2007) 249-253.

13) M. Tachikawa and T. Itoh: J. High Temp. Soc. 36 (2010) 80-84 [in Japanese].

14) T. Itoh and M. Tachikawa: Mater. Res. Soc. Symp. Proc., Vol. 1314, (Cambridge University Press, 2011) pp. mrsf10-1314-1108-18 (6pp). 